\title{
Fluoride - an adjunctive therapeutic agent for periodontal disease? Evidence from a cross-sectional study
}

\author{
Santhosh Kumar ${ }^{1}$, Jyothi Sharma ${ }^{1}$, Prabu Duraiswamy ${ }^{2}$, Suhas Kulkarni ${ }^{3}$ \\ ${ }^{1}$ MDS, Post graduate student Department Preventive and Community Dentistry Darshan Dental College and Hospital Udaipur, \\ India \\ ${ }^{2}$ MDS, Associate professor Department Preventive and Community Dentistry Darshan Dental College and Hospital Udaipur, \\ India \\ ${ }^{3}$ MDS, Professor and Head Of Department Department Preventive and Community Dentistry Darshan Dental College and \\ Hospital Udaipur, India
}

Correspondence:

Department of preventive and community dentistry

Darshan Dental College and Hospital

Udaipur. Rajasthan

India. 313001

santosh_dentist@yahoo.com

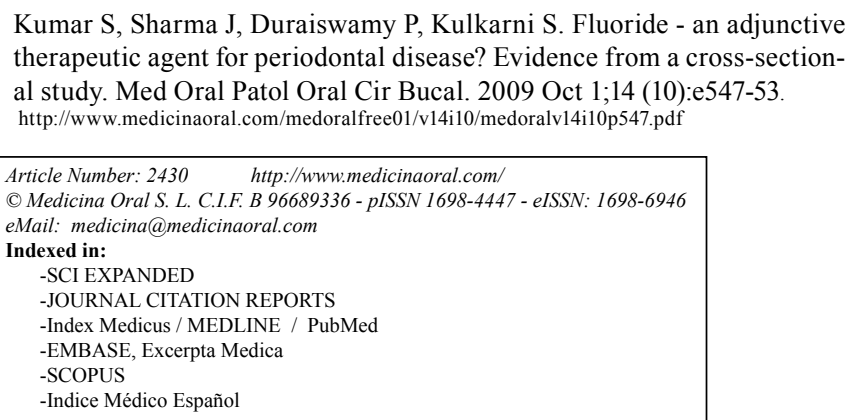

\begin{abstract}
Objective: To assess the influence of the water fluoride level on periodontal status, by determining the periodontal health status of subjects residing in low, optimum and high fluoride areas. Study design: A cross sectional survey was carried out on 967 adults aged 35-44 years old, from the Udaipur district of India. A stratified cluster random sampling technique was implemented in order to collect a representative sample from low $(<0.6 \mathrm{ppm})$, optimum $(0.6-1.2 \mathrm{ppm})$ and high fluoride ( $>3 \mathrm{ppm})$ areas, based on the fluoride concentration in drinking water. Periodontal status was assessed in accordance to WHO criteria. The Chi-square test was used to compare proportions, and logistic regression analysis was used to determine the contribution of water fluoride levels to periodontal disease. Results: Those residing in areas of low fluoride levels were more likely to present periodontal pockets than those living in high fluoride areas $1.3(95 \%$ CI $1.11 \pm 1.86)$. Subjects living in areas of low fluoride were noted to have a higher risk of periodontal attachment loss of more than $8 \mathrm{~mm}(\mathrm{OR}=1.94,95 \%$ CI $1.67 \pm 3.85)$. The risk for presence of periodontal pockets and attachment loss of more than $8 \mathrm{~mm}$ increased by 1.17 (95\% CI 1.02 \pm 1.69 ) and 1.59 (95\% CI 1.27 \pm 3.29$)$ respectively for those residing in areas of optimum fluoride levels. Deep periodontal pockets were more prevalent (6.3\%) among those residing in areas of low fluoride, followed by optimum (5.2\%) and high (3.1\%). Conclusions: As the fluoride concentrations increased, the prevalence of shallow and deep periodontal pockets decreased. The severity of periodontal disease was significantly associated with fluoride levels, with cases of loss of attachment gradually decreasing when moving from low fluoride areas to high fluoride areas. It appears that longitudinal studies need to be conducted in order to ascertain the benefits; and microbiological analysis of dental plaque and periodontium should be carried out in order to confirm the effects of fluoride on periodontal conditions.
\end{abstract}

Key words: Adjunctive therapy, periodontal disease, water fluoridation. 


\section{Introduction}

Periodontal disease is one of the two major dental diseases that affect human populations worldwide at high prevalence rates (1) and it was observed that the prevalence of periodontal disease is greater in developing countries than the industrialized ones (2).

The role of personal risk factors such as poor life style and negative psychosocial conditions has been said to play an important role in the etiology of adult periodontitis (3).

Moreover, the inhibition of bone resorption by fluoride in a variety of model systems suggests that a high $\mathrm{F}$ intake might protect against alveolar bone loss in periodontal disease (4).

Zimmerman et al. (5) compared the oral health status of residents of two areas with high $(8 \mathrm{ppm})$ and low $(0.4 \mathrm{ppm})$ fluoride concentration and concluded that no significant difference was found between the regions for amount of gingivitis and alveolar bone resorption.

Russell (6) and Englander (7) both detected a lower percentage of adults with pockets in the fluoridated community than that of the non fluoridated community.

Fluoridated drinking water may affect periodontal disease through direct or indirect mechanisms (8). Direct effects may occur if fluoridation decreases the amount of adherence of plaque or if fluorosed teeth inhibit plaque (8).

Fluoridation may also affect indirectly (8) on the periodontal tissue by reducing caries and the amount of restorative treatment. If fluoridation reduces interproximal caries, fewer restorations may be placed on smooth tooth surfaces, resulting in less subgingival calculus. Similarly, if fluoridation decreases interproximal restorations, fewer overhangs may occur, this may reduce attachment loss, pocket depth and the number of interproximal bleeding sites. Fluoridation may also reduce the amount of recession from fillings placed on buccal surfaces.

Though, there are contradictory results from various epidemiological studies which assessed the difference between periodontal conditions of fluoride and non-fluoride areas, many previous studies observed high levels of gingival inflammation in endemic fluorosed areas than those of non fluorosed ones.

Developing countries in Asia and Africa which are undergoing a social change have shown an upward trend in the severity and prevalence of dental diseases.

Previous epidemiological studies on the presence and severity of periodontal disease have been reported that periodontal health is worst in developing countries than in industrialized ones (2). Water fluoride if proven effective in periodontal disease prevention would be a cheap measure to implement in developing countries. Periodontal disease which is prevalent in more than $90 \%$ Indian population is responsible for the high dental morbidity in the country (9). Moreover, it is reported that $85 \%$ of children suffer from periodontal disease at a point in time and $89.6 \%$ adults of $35-44$ year age group are afflicted with the diseases (10).

Various studies have proved the efficacy of fluoride both topical and systemic on the dental caries prevalence whereas regarding the periodontal disease the available data to date is not adequate to prove or disprove that fluoride reduces or prevents periodontal disease (11). The present study is one such attempt to assess the influence of water fluoride level on periodontal status by determining the periodontal health status of subjects residing in low, optimum and high fluoride areas.

\section{Material and Methods}

Study area pertained to Udaipur district of India and the final sample size accounted to 967 permanent residents aged between 35 to 44 years.

Multi-stage stratified cluster random sampling procedure was executed to collect the representative sample. At first stage, all the panchayat samitis in the Udaipur district were stratified as low $(<0.6 \mathrm{ppm})$, optimum $(0.6-1.2 \mathrm{ppm})$ and high fluoride ( $>3 \mathrm{ppm})$ based on the fluoride concentrations in drinking water (data from ground water department, Udaipur district). At second stage, one panchayat samiti from each stratum was selected randomly and subsequently, from each chosen panchayat samiti three villages were selected randomly. Thus a total of nine villages were selected in the district. From each of the selected village, all the households were covered, starting from northeast corner of the villages. Fluoride concentration in water of a common well which was used by villagers was assessed using ion specific electrode method.

Men who were permanent residents and those who were present at home on the days of the survey between the ages of 35 to 44 years were included in the study and exclusion criterion comprised of the uncooperative (32 subjects), chronic ill (23 subjects), tobacco consumers (212 subjects) and those who had undergone any type of periodontal surgeries or oral prophylaxis in the last one year (4 individuals) and those subjects who consumed water from the source other than the one which was assessed for fluoride content (22 individuals) to avoid confounding results for periodontal status assessment.

Initially both male and female subjects in the age group $35-44$ years were invited to participate. However, the acceptance of such a procedure by the females in the first village was extremely low when oral examination was attempted by the male investigator. Hence the study sample comprised only male subjects who were at home when the investigator visited.

Ethical approval for conducting the study was availed from ethical committee for research of Darshan Dental College and Hospital. Study was conducted during March 2008 to may 2008. 
Clinical examination was done by a single examiner under adequate illumination and clinical data were collected on periodontal status by means of Community Periodontal Index (CPI) and Loss of Attachment (LA) as described by the World Health Organization. The examiner was blind regarding the water fluoride level in various regions.

Before conducting the survey, the investigator was trained and calibrated by an expert examiner. Moreover, in order to minimize the intra examiner variability, $10 \%$ subjects who underwent the clinical examinations were randomly selected in each village for repeated examination. Kappa co-efficient for intra-examiner variability was found to be $93 \%$ for CPI and $86 \%$ for LA.

Community Periodontal Index (1) (CPI) was used to record the periodontal condition which is performed using a mouth mirror and CPI probe. Information on loss of attachment (14) was collected from index teeth based on WHO criteria using CPI probe in order to obtain an estimate of the lifetime accumulated destruction of the periodontal attachment. The scores for loss of attachment ranged from 0 (attachment loss of $0-3 \mathrm{~mm}$ ) to 4 (attachment loss of $12 \mathrm{~mm}$ or more; CEJ beyond $11.5 \mathrm{~mm}$ ring).

Data collected was entered into the spreadsheets; statistical package for social sciences (SPSS) version 15.0 was used for data analysis. As the sampling procedure was complex, "Complex Sample" module in SPSS was used for statistical analysis.
Chi-square analysis was executed to assess the difference in the proportions of populations from low, medium and high fluoride areas with various periodontal disease indicators.

Multiple logistic regression analysis was conducted to assess if the water fluoride level significantly predicted for the occurrence of periodontal disease and attachment loss. To facilitate logistic regression analysis, dependent variables (each category of CPI and LA) were dichotomized; healthy periodontium vs bleeding, healthy periodontium vs calculus, healthy periodontium vs shallow pockets and healthy periodontium vs deep pockets. Similarly all the categories of loss of attachment were analysed.

The categories in each independent variable comprised of water fluoride level (high, optimum and low), age of the subjects ( $35-39$ years old and $40-44$ years old), brushing frequency (at least once a day and less than once a day), dental visiting habits (never visited in past one year and visited dentist in past one year).

The effect of each independent variable was assessed adjusting for that of all others in the model.

\section{Results}

The results showed that the proportions of subjects who reported periodontal pockets decreased steadily with the water fluoride level and significant difference was observed $(\mathrm{P}<0.05)$ for various periodontal disease indicators between the residents of low, optimum and high water fluoride area (Fig. 1).

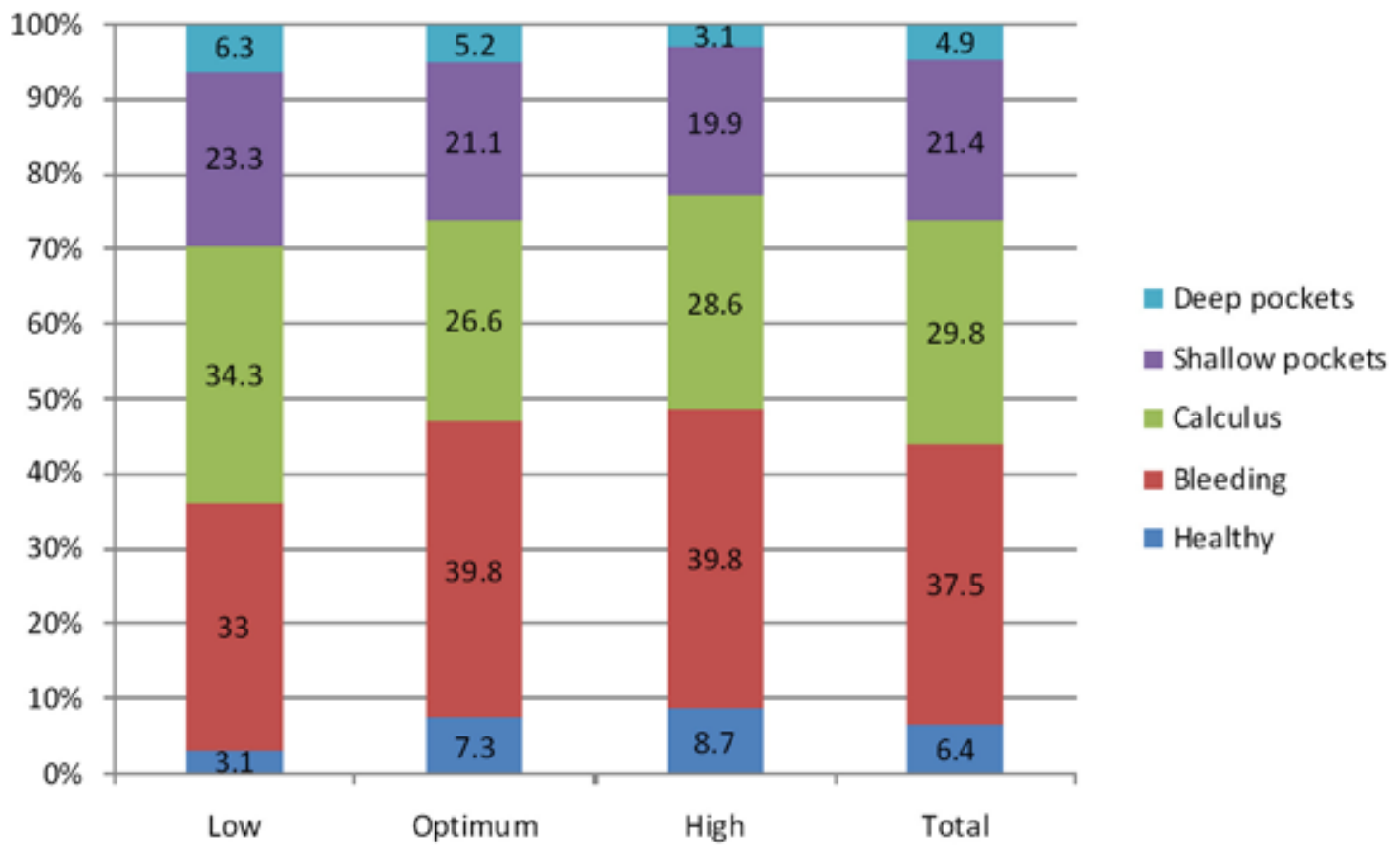

Fig. 1. Prevalence of periodontal disease among the residents of Udaipur district according to water fluoride level. 


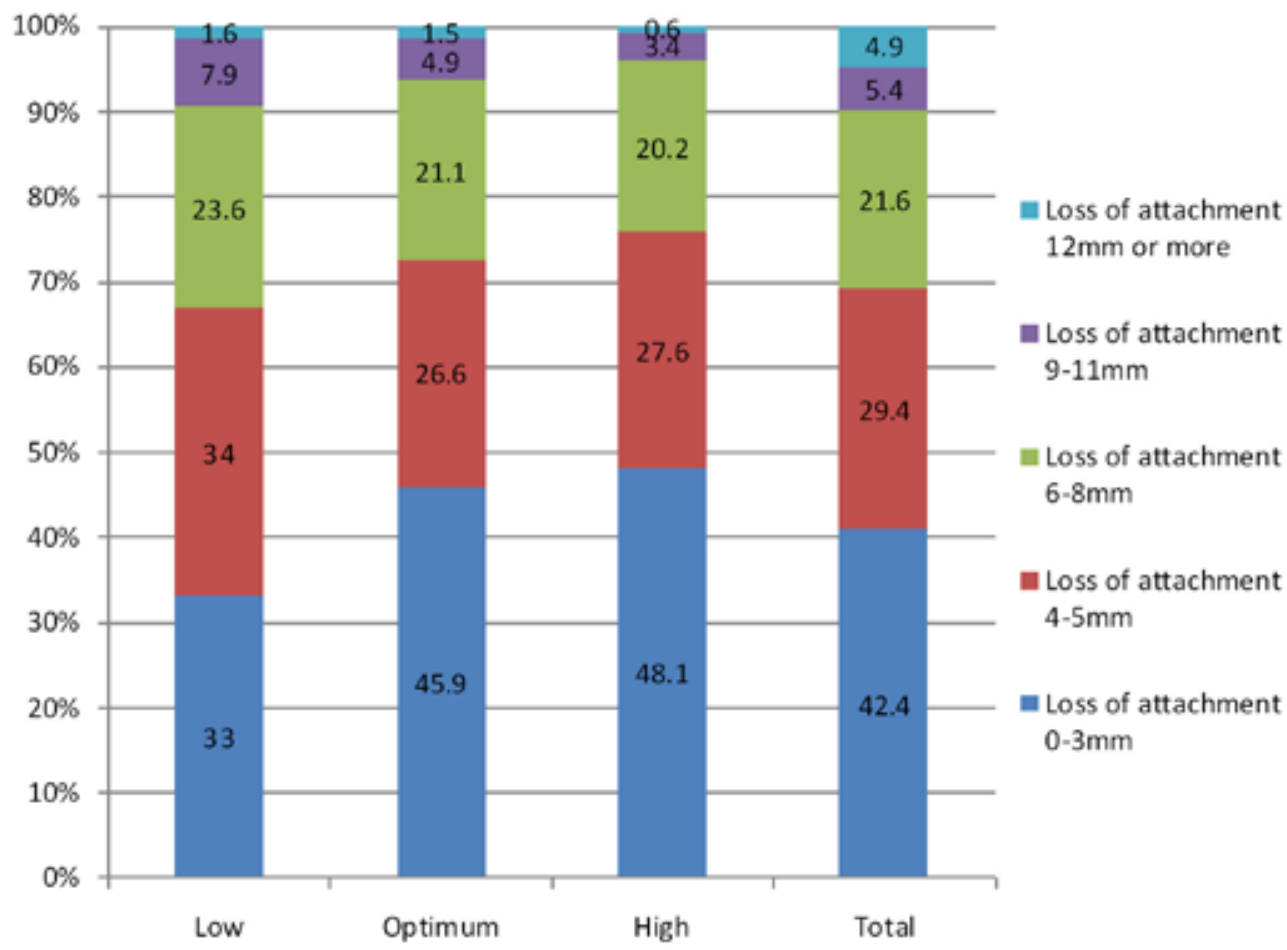

Fig. 2. Loss of Attachment status among study subjects at low, optimum and high fluoride areas of Udaipur district.

Table 1. Logistic regression analysis with various categories of CPI as dependent variables (healthy periodontium Vs bleeding or calculus or pockets) and water fluoride level, age, frequency of cleaning teeth, dental visiting practices as independent variables.

\begin{tabular}{|c|c|c|c|c|}
\hline & Bleeding & Calculus & Shallow pockets & Deep pockets \\
\hline Independent variables & Odds ratio $(95 \% \mathrm{CI})$ & Odds ratio $(95 \% \mathrm{CI})$ & Odds ratio $(95 \% \mathrm{CI})$ & Odds ratio $(95 \% \mathrm{CI})$ \\
\hline \multicolumn{5}{|l|}{ Water fluoride level } \\
\hline High & $1.0 \dagger$ & 1.0 & $1.0 \dagger$ & 1.0 \\
\hline Optimum & $0.58(0.14-0.89)$ & $0.94(0.42-1.31)$ & $1.24(1.11-1.84)$ & $1.10(1.02-1.94)$ \\
\hline Low & $0.32(0.09-0.56)$ & $1.79(0.88-2.04)$ & $1.99(1.28-2.32)$ & $2.66(1.88-2.85)$ \\
\hline \multicolumn{5}{|l|}{ Age } \\
\hline $35-39$ & 1.0 & 1.0 & $1.0 \dagger$ & $1.0 \dagger$ \\
\hline $40-44$ & $0.40(0.16-1.01)$ & $0.21(0.08-0.53)$ & $1.14(1.05-1.38)$ & $1.88(1.12-3.72)$ \\
\hline \multicolumn{5}{|l|}{ Tooth cleaning frequency } \\
\hline At least once a day & $1.0 \dagger$ & $1.0 \dagger$ & 1.0 & 1.0 \\
\hline Less than once a day & $1.48(1.03-1.82)$ & $1.12(1.08-1.34)$ & $2.18(1.43-2.82)$ & $1.12(1.08-1.34)$ \\
\hline \multicolumn{5}{|l|}{ Dental visiting practices } \\
\hline Visited dentist in past one year & $1.0 \dagger$ & $1.0 \dagger$ & $1.0 \dagger$ & 1.0 \\
\hline Never visited a dentist & $1.30(1.15-1.92)$ & $1.77(1.31-1.91)$ & $2.64(1.83-3.31)$ & $1.87(0.60-2.29)$ \\
\hline
\end{tabular}

$\dagger \mathrm{p}<0.05$ 
Table 2. Logistic regression analysis with various categories of LA (Loss of attachment) as dependent variable (LA 0-3mmVs LA 4- 5mm or LA $6-8 \mathrm{~mm}$ or LA $9-11 \mathrm{~mm}$ or LA $12 \mathrm{~mm}$ and more) and water fluoride level, age, frequency of cleaning teeth, dental visiting practices as independent variables.

\begin{tabular}{|c|c|c|c|c|}
\hline & LA 4 -5 mm & LA 6 -8 mm & LA 9 -11mm & LA $12 \mathrm{~mm}$ or more \\
\hline Independent variables & Odds ratio $(95 \% \mathrm{CI})$ & Odds ratio $(95 \% \mathrm{CI})$ & Odds ratio $(95 \% \mathrm{CI})$ & Odds ratio $(95 \% \mathrm{CI})$ \\
\hline \multicolumn{5}{|l|}{ Water fluoride level } \\
\hline High & $1.0 \dagger$ & $1.0 \dagger$ & $1.0 \dagger$ & $1.0 \dagger$ \\
\hline Optimum & $0.97(0.36-0.95)$ & $0.64(0.42-0.88)$ & $1.95(1.25-3.48)$ & $1.21(1.05-1.83)$ \\
\hline Low & $0.32(0.09-0.56)$ & $0.89(0.26-0.97)$ & $2.26(1.48-4.64)$ & $3.97(1.62-5.07)$ \\
\hline \multicolumn{5}{|l|}{ Age } \\
\hline $35-39$ & $1.0 \dagger$ & 1.0 & $1.0 \dagger$ & 1.0 \\
\hline $40-44$ & $0.52(0.35-0.78)$ & $0.37(0.24-0.58)$ & $1.12(1.04-1.34)$ & $1.52(0.55-1.97)$ \\
\hline \multicolumn{5}{|l|}{ Tooth cleaning frequency } \\
\hline At least once a day & 1.0 & 1.0 & $1.0 \dagger$ & $1.0 \dagger$ \\
\hline Less than once a day & $0.96(0.44-1.81)$ & $1.77(0.81-3.85)$ & $2.23(1.71-3.16)$ & $2.62(1.41-5.07)$ \\
\hline \multicolumn{5}{|l|}{ Dental visiting practices } \\
\hline Visited dentist in past one year & 1.0 & $1.0 \dagger$ & $1.0 \dagger$ & 1.0 \\
\hline Never visited a dentist & $0.73(0.58-2.91)$ & $3.24(1.46-7.21)$ & $3.87(1.74-5.13)$ & $2.10(0.69-7.27)$ \\
\hline
\end{tabular}

$\dagger \mathrm{p}<0.05$

The highest percentage of subjects with shallow and deep pockets were found in the low water fluoride area, being $23.3 \%$ and $6.3 \%$ respectively. Deep periodontal pockets were more prevalent $(6.3 \%)$ among the subjects of low fluoride region followed by optimum (5.2\%) and high (3.1\%).

There was no definite trend for the distribution of subjects with bleeding and calculus among the three localities with different water fluoride level.

It is evident from figure 2 that $33 \%$ of the residents presented a loss of attachment of $0-3 \mathrm{~mm}$ whereas it was $48.1 \%$ among the high fluoride area subjects.

There was a definite trend for all the LOA scores with the percentage of subjects decreasing as the water fluoride level increased.

$7.9 \%$ subjects in the low fluoride area, $4.9 \%$ subjects in the optimum fluoride area and 3.4\% subjects in the high fluoride area had LOA scores of 3. Similar trend was observed for attachment loss of more than $12 \mathrm{~mm}$ with $1.6 \%, 1.5 \%$ and $0.6 \%$ subjects belonging to low, optimum and moderate fluoride areas respectively.

Chi square analysis revealed a statistically significant difference between the LOA scores $(\mathrm{p}<0.05)$.

Logistic regression analysis was employed to determine the effect of water fluoride level, age, frequency of cleaning teeth and dental visiting practices of the study population on the occurrence of various periodontal disease indicators including loss of attachment. Table 1 illustrates the association between water fluoride level and occurrence of periodontal disease indicators which was assessed by CPI. The odds ratio of presenting shallow or deep periodontal pockets increased gradually as the water fluoride level decreased with low fluoride area dwellers being more liable for presence of periodontal pockets than high fluoride area inhabitants $(1.99,95 \%)$ CI $1.11-1.86$ for shallow pockets and 2.66, $95 \%$ CI $1.88-2.85)$. However, subjects from high fluoride areas were more liable for bleeding and calculus than subjects residing in low fluoride areas.

Age exerted a significant influence for the presence of pockets with subjects belonging to $40-44$ years age group being at more risk for presence of pockets than those belonging to the younger age group. Furthermore, tooth cleaning frequency and dental visiting practices had an effect on occurrence of periodontal disease with subjects brushing at least once a day and who never visited a dentist more prone for presenting bleeding, calculus and pockets.

Table 2 illustrates the influence of various independent variables on the occurrence of periodontal attachment loss. Subjects had a higher risk of having periodontal attachment loss if they lived in low fluoride area $(\mathrm{OR}=$ $1.94,95 \%$ CI $1.67-3.85)$. The risk for periodontal attachment loss of $9-11 \mathrm{~mm}$ and $12 \mathrm{~mm}$ or more increased 
by 2.26 (95\% CI $1.48-4.64)$ and 3.97 (95\% CI 1.62 -5.07 ) respectively for subjects residing in low fluoride areas. Moreover, the subjects in the $40-44$ year old age group were more liable for attachment loss of more than $8 \mathrm{~mm}$ than the younger age group. Even tooth cleaning and dental visiting practices had an influence on the severity of attachment loss.

\section{Discussion}

The relative roles of the various suspected etiologic factors in periodontal disease have been investigated by means of epidemiologic surveys and clinical studies but there are no studies executed to assess the effect of water fluoride concentration on periodontal disease, though there were few studies in the past they have not succeeded in confirming the relationship between periodontal disease and community water fluoride level.

The present study attempted to assess the periodontal health status of subjects residing in low, optimum and high fluoride areas measured by Community periodontal index and Loss of attachment.

The results of the present study could not be compared validly with the past studies due to variations in the study designs, age group, gender, indices undertaken and methodology executed. The subjects in each locality selected in the present study were similarly distributed according to oral hygiene habits, ethnicity, socioeconomic status and education hence, these factors could not have contributed to the periodontal differences observed. However, the study is limited by the fact that only one panchayat samiti (primary sampling unit) was selected from each strata due to the inadequate resources available.

There are conflicting reports from literature regarding the influence of water fluoride on the periodontal status till date with Zimmermann et al. (5) observing no significant differences in gingivitis, radiographic bone loss and supragingival calculus between the subjects of Barlett (8ppm) and Cameron (0.5ppm) in Texas, whereas, Murray (12) observed that gingivitis and gingival recession (loss of attachment) in residents of Hartlepool (1.2ppm-2.0ppm) was more prevalent than York (0.150.2 ), even in our study prevalence of gingival bleeding in the low $(33 \%)$ water fluoride area was lower than the moderate $(39.8 \%)$ and high fluoride area $(39.8 \%)$ whereas in 10-year summaries of the fluoridation study at Newburgh, it was reported that there was slightly but significantly more gingivitis in children of Kingston, the control city, than in children of Newburgh who had used fluoridated water for from seven to 10 years (13). While, Reddy et al. (15) observed that the prevalence and severity of periodontitis was not influenced by fluoride content.

Another study (7) compared the periodontal status between two localities with fluoride level more than 1.2 ppm and $0.1 \mathrm{pm}$ respectively using Russell's periodontal index. It was found that adults in the high fluoride community had a lower percentage of pockets than those residing in low fluoride areas whereas no difference was observed for plaque and calculus. Moreover, the mean PI of 0.69 for natives of Aurora (0.1 ppm) was lower than the score of 0.84 in Rockford (1.2 ppm), similar results were observed in the present study where low fluoride area dwellers were more liable for presence of periodontal pockets than high fluoride area inhabitants. Russell (6) observed that $23.4 \%$ of the subjects aged 35 44 year old of Colorado Springs (2.5 ppm F) presented periodontal pockets whereas $56 \%$ subjects from Boulder (fluoride free) had pockets which implies that there were $32.6 \%$ more subjects in fluoride free locality, this is in accordance with the present study but the difference in the prevalence of pockets in low and high fluoridated areas was $6.8 \%$. The probable reason for this observation may be due to several reasons firstly, periodontal disease has declined over the past decades, which may have reduced the magnitude of the association between fluoridation exposure and periodontal pockets. The second reason may be the difference in water fluoride content in the earlier study and the present study.

Numerous animal studies (4) have produced results ranging from an inhibition by $\mathrm{F}$ of alveolar bone loss to a slight increase in alveolar bone loss. In one animal study by Messer et al. (4), no significant differences in alveolar bone loss were observed between the mice that were fed a standard low F (Fluoride) diet containing 0.1$0.5 \mathrm{ppm} F$ with deionized water (low F group) and water containing $50 \mathrm{ppm} \mathrm{F}$.

A study (12) from India that assessed the periodontal status in dental fluorosis subjects observed that as the degree of fluorosis increased severity of gingivitis reduced and periodontitis increased

The results of the past study are limited as no attempt was made to control the effect of age and gender.

Grembowski et al. (8) observed that relative to adults with no fluoride exposure, continuous lifetime exposure reduces the probability of attachment loss from 0.87 to 0.72 ; this is in accordance with the present study. Although in the present study the subjects had a higher risk of having periodontal attachment loss of more than $8 \mathrm{~mm}$ if they lived in low fluoride area, direct comparisons are not possible as the attachment loss in the earlier study was a binary measure (present or absent).

Most of the past studies assessed the difference in prevalence and severity of periodontal disease between the optimally fluoridated areas and non fluoridated areas but no studies observed the dose response relationship of fluoride and periodontal status; it was evident from the results of our study that as the fluoride level in drinking water increased the periodontal status improved.

The scientific basis for the beneficial effects of fluoride 
on the periodontal tissues has been studied by many authors, among which Bjorvatn et al. (14) found that $1 \%$ sodium fluoride reduced root resorption among mice. They ascribed this result to the formation of fluoroapatite or to the effect on osteoclasts.

While, another study (15) reported that the conversion of hydroxyapatite to fluoroapatite in cementum in high fluoride area renders the periodontium resistant to breakdown.

Alternatively fluoride may indirectly influence the periodontal status by definite decrease in plaque accumulation from low to high fluoride areas as reported by Anuradha et al. (16).

Moreover, the evidence of antibacterial effect of fluoride was confirmed by many previous studies, Rö lla et al. (17) proved that the presence of fluoride results in decreased absorption of protein to hydroxyapatite in addition to decreased absorption of bacteria, Yoon and Berry (18) observed that fluoride in various forms were effective in decreasing count of Actinomyces, Yoon NA and Newman (18) demonstrated the effects of fluoride on Bacteroides melaninogenicus subspecies and Bacteroides asaccharolyticus, the inhibition of glycoloytic enzyme enolase by Hamilton (18) are the likely mechanisms where in inflammatory aspect of periodontal disease is dealt with. It is possible that one or more of these mechanisms may explain the results observed in the present study.

Moreover, it was observed that subjects in the older age group were at more risk for presence of pockets and attachment loss of more than $8 \mathrm{~mm}$ than those belonging to the younger age group, previous studies $(19,20)$ have shown that prevalence and severity of periodontal disease increases with age.

The persons who cleaned their teeth less than once a day tended to exhibit periodontal attachment loss more than the subjects who cleaned at least once a day this is in agreement with the study by Amarasena et al. (20) which reported the association of increasing frequency of tooth cleaning with reduced levels of plaque index, gingival index and loss of attachment. The periodontal status deteriorated among the subjects who never visited dentist, the rational explanation for this may be the accumulated disease among those subjects.

The findings of the present study are motivating but they do not provide a sufficient basis for claims that lifelong consumption of fluoridated water has a direct protective effect upon periodontal status. The results of the present study revealed that as the fluoride concentrations increased the prevalence of shallow and deep periodontal pockets decreased and gingival bleeding was more prevalent in optimum and high fluoride areas than low fluoride localities. Severity of the periodontal diseases was significantly associated with fluoride levels with loss of attachment gradually decreasing from low fluoride area to high fluoride area. It appears there is a need for execution of longitudinal studies to ascertain the benefits of this adjunct therapeutic agent. Microbiological analyses of dental plaque and periodontium in order to confirm the effects of fluoride on periodontal conditions are even required.

\section{References}

1. Petersen PE, Ogawa H. Strengthening the prevention of periodontal disease: the WHO approach. J Periodontol. 2005;76:2187-93.

2. Pilot T. The periodontal disease problem. A comparison between industrialised and developing countries. Int Dent J. 1998;48:221-32.

3. Clarke NG, Hirsch RS. Personal risk factors for generalized periodontitis. J Clin Periodontol. 1995;22:136-45.

4. Messer HH, Singer L, Ophaug R. Fluoride intake and alveolar bone loss in periodontal disease-susceptible mice. J Periodontal Res. 1983;18:82-5.

5. Zimmermann ER, Leone NC, Arnold FA Jr. Oral aspects of excessive fluorides in a water supply. J Am Dent Assoc. 1955;50:272-7.

6. Russell AL. Fluoride domestic water and periodontal disease. Am J Public Health Nations Health. 1957;47:688-94.

7. Englander HR, Kesel RG, Gupta OP. The Aurora-Rockford, ill., study. II. Effect of natural fluoride on the periodontal health of adults. Am J Public Health Nations Health. 1963;53:1233-42.

8. Grembowski D, Fiset L, Spadafora A, Milgrom P. Fluoridation effects on periodontal disease among adults. J Periodontal Res. 1993;28:166-72.

9. Mistry KM. The changing pattern of oral disease in India--the need for a preventive approach. J Indian Dent Assoc. 1983;55:38793.

10. Kumar S, Dagli RJ, Chandrakant D, Prabu D, Suhas K. Periodontal status of green marble mine labourers in Kesariyaji, Rajasthan, India. Oral Health Prev Dent. 2008;6:217-21.

11. Suomi JD. Prevention and control of periodontal disease. J Am Dent Assoc. 1971;83:1271-87.

12. Vandana KL, Reddy MS. Assessment of periodontal status in dental fluorosis subjects using community periodontal index of treatment needs. Indian J Dent Res. 2007;18:67-71.

13. Ast DB, Schlesinger ER. The conclusion of a ten-year study of water fluoridation. Am J Public Health Nations Health. 1956;46:26571.

14. Bjorvatn K, Massler M. Effect of fluorides on root resorption in replanted rat molars. Acta Odontol Scand. 1971;29:17-29.

15. Reddy J, Grobler SR. The relationship of the periodontal status to fluoride levels of alveolar bone and tooth roots. J Clin Periodontol. 1988;15:217-21.

16. Anuradha KP, Chadrashekar J, Ramesh N. Prevalence of periodontal disease in endemically flourosed areas of Davangere Taluk, India. Indian J Dent Res. 2002;13:15-9.

17. Rölla G, Melsen B. Desorption of protein and bacteria from hydroxyapatite by fluoride and monofluorophosphate. Caries Res. 1975;9:66-73.

18. Mandell RL. Sodium fluoride susceptibilities of suspected periodontopathic bacteria. J Dent Res. 1983;62:706-8.

19. Albandar JM. Periodontal diseases in North America. Periodontol 2000. 2002;29:31-69.

20. Amarasena N, Ekanayaka AN, Herath L, Miyazaki H. Tobacco use and oral hygiene as risk indicators for periodontitis. Community Dent Oral Epidemiol. 2002;30:115-23. 